\title{
The effect of emotional intensity on responses to joint attention in preschoolers with an autism spectrum disorder
}

\author{
Martina Franchini ${ }^{1,2}$, Bronwyn Glaser ${ }^{1}$, Edouard Gentaz ${ }^{2}$, Hilary Wood ${ }^{1}$, Stephan Eliez ${ }^{1,3}$, Marie Schaer ${ }^{1,4}$
}

\begin{abstract}
${ }^{1}$ Behavioral Psychiatry and Neuroimaging Lab, University of Geneva, 1 Rue David-Dufour, Geneva, Switzerland ${ }^{2}$ Sensorimotor, Affective and Social Development Unit, 40 Bd du Pont-d'Arve, 1205 Geneva, Switzerland ${ }^{3}$ Department of Medical Genetic, Geneva University Medical School, 1 Rue Michel-Servet, 1206 Geneva, Switzerland

${ }^{4}$ Stanford Cognitive \& Systems Neuroscience Laboratory, Stanford University, 1070 Arastradero Rd. Suite 220, Palo Alto, USA
\end{abstract}

Corresponding author: Martina Franchini, martina.franchini@unige.ch,

Behavioral Psychiatry and Neuroimaging Lab

1, Rue David-Dufour

1203 Geneva (Switzerland)

$+41223886749$ 


\begin{abstract}
Background

Responding to joint attention (RJA) is reduced in young children with ASD despite being vital to the early development of social communication. For this reason, RJA is a frequent target in early intervention programs. Clinical guidelines suggest that exaggerating facial expressions or gestural pointing may improve RJA in children with autism by clarifying the meaning of the gaze or gesture. In the current study, we investigate the effect of intensity of emotional expression on RJA in preschoolers with ASD.
\end{abstract}

Method

Twenty-five preschoolers with ASD and 21 typically developing peers were administered a specifically designed eyetracking task in which an actor directs attention to a moving object by looking at it with an expression that was either neutral, mildly surprised or intensely surprised. In a supplementary condition, gestural pointing was accompanied by a neutral gaze shift.

Results

Commensurate with previous studies, children with ASD oriented less to the face of the actor and were less responsive to RJA when compared to their typically developing peers. However, children with autism differed in their response to RJA according to the different task conditions. They demonstrated more frequent responses to RJA when the actor's facial expression was either intense or supported by gestural pointing.

\title{
Conclusions
}

We report initial evidence for the importance of clarifying content cues in order to help preschoolers with autism adhere to receptive joint attention (RJA) opportunities. Our findings inform knowledge about early treatment and attention to emotional expressions in autism and improve our understanding of how early interventions may work to increase RJA in young children with ASD.

\section{Keywords}

Autism Spectrum Disorders, Eye-tracking, Response to joint attention (RJA), Emotional expressions, Gestural pointing 


\section{Introduction}

Joint attention (JA) represents the ability to share attention and refers to one of our earliest communication experiences as young children (Mundy, Sigman, \& Kasari, 1990; Toth, Munson, Meltzoff, \& Dawson, 2006). Young children with Autism Spectrum Disorders (ASD) demonstrate JA behaviors that are reduced both in quantity and quality compared to typically developing (TD) children (Mundy, Sigman, Ungerer, \& Sherman, 1986; Navab, Gillespie Lynch, Johnson, Sigman, \& Hutman, 2012). Indeed, reduced JA is one of the most consistent features of ASD and even serves as a diagnostic criterion for the disorder (Lord et al., 2000). JA behaviors can be expressive (initiation of joint attention behaviors, IJA) or receptive (responding to joint attention behaviors, RJA) by nature. In this paper, we will mostly focus on RJA, which refers to "infants' ability to follow the direction of the gaze and gestures of others to share a common point of reference" (Mundy et al., 2007, p. 269). RJA increases shared visual attention to objects (for a review see Frischen, Bayliss \& Tipper, 2007), which provides learning opportunities for communication (e.g. word designation) in both TD children (Brooks \& Meltzoff, 2008) and children with ASD (Murray et al., 2008, Whalen \& Schreibman, 2006; Jones, Carr \& Feeley, 2006).

TD infants inherently direct their attention toward facial expressions, which represent socially salient cues for them (for a review see Frischen, Bayliss \& Tipper, 2007). This attention to faces in 2-3 month-old TD infants supports the development of JA skills through dyadic interactions between the child and his/her caretaker (Leekam \& Ramsden, 2006; for a review see Aubineau, Le Driant, \& Vandromme, 2014). Accordingly, when an infant orients himself to his socially relevant surrounding environment, he accesses opportunities to develop attention-sharing skills, such as JA. This prevalent early interest for the social world appears to be altered in autism (Chevallier, Kohls, Troiani, Brodkin, \& Schultz, 2012; Dawson, Bernier, \& Ring, 2012; Dawson et al., 2004). Infants at high risk for ASD demonstrated attention to the eyes at 2 months of age, but their attention had declined by 6 months of age (Jones \& Klin, 2013). And there is evidence that it does not rebound; attention to eyes and faces in toddlers with ASD is reduced compared to their TD peers (Chawarska, Macari, \& Shic, 2012; Osterling, Dawson, \& Munson, 2002).

Given the key role of JA in the development of communication skills in children with ASD (Murray et al., 2008, Whalen \& Schreibman, 2006; Jones, Carr \& Feeley, 2006), attention sharing behaviors represent a critical target for early intervention programs designed for young children with autism (Charman et al., 2003; Jones \& Carr, 2004; Jones, Carr, \& Feeley, 2006; Rogers et al., 2012). Practice coordinating gazing and pointing when initiating JA with ASD children can improve their ability to respond to JA overtures (Carpenter, Pennington, \& Rogers, 2002; Claes Hofsten, Dahlström, \& Fredriksson, 2005). Exaggerating facial expressions and gestures, another early intervention technique, can make the behavioral content of the gaze and/or gesture clearer, thus helping RJA (Ingersoll \& Dvortcsak, 2006; Taylor \& Hoch, 2008; Mead \& Mataric, 2010; Rogers \& Dawson, 2010). Finally, several theoretical papers and 
books on ASD interventions suggest that expressing surprise in naturalistic situations may cue RJA behaviors (LeComer, 2009; Mastrangelo, 2009).

Several studies show the importance of emotional facial expressions to gaze shift in RJA. For example, work on primates suggests that detection of facial expressions contributes to the development of RJA by facilitating early gaze-following (Teufel, Gutmann, Pirow, \& Fischer, 2010). Growing evidence from adult human studies also suggests that attention and correct detection of emotional facial expressions may play a role to accurately responding to other people's gaze shifting (Bayliss, Frischen, Fenske, \& Tipper, 2007; Bayliss, Paul, Cannon, \& Tipper, 2006; Itier \& Batty, 2009; Soussignan, Schaal, Boulanger, Garcia, \& Jiang, 2015). Studies on TD children similarly suggest that toward the end of the first year of life, negative facial expressions (e.g. angry faces) already attract eye gaze (Niedzwiecka \& Tomalsi; Hoehl et al., 2008). Furthermore, social appraisal studies (e.g. Mumenthaler et Sander, 2012) suggest that other people's emotional reactions influence the way individuals appraise the same event. In a study using subliminal emotional faces looking at a second emotional face, the authors demonstrate that socio-affective inference can have a major influence on the way that we perceive emotions (Mumenthaler $\&$ Sander, 2015). Given the importance of emotional expression in events of social reference, as well as the aforementioned clinical observations that support the importance of emotional expressions to RJA, we postulate that intense expressions could enhance attention to faces in young children with or without ASD and help them to better recognize and respond to a JA behavior initiated by another person. To the best of our knowledge, previous studies have not investigated the effect of emotional intensity on attention to faces or RJA in young children with ASD.

Eye-tracking provides a unique opportunity to quantify RJA in a precise and standardized way (Chawarska et al., 2012; Chawarska, Klin, \& Volkmar, 2003; Falck-Ytter, Fernell, Hedvall, Hofsten, \& Gillberg, 2012; Navab et al., 2012). Measuring gaze patterns while watching video clips containing examples of joint attention behavior makes it possible to quantify time spent on a face (Chawarska et al., 2012; Chawarska, Klin, \& Volkmar, 2003) or the number of gaze shift between a face and a referenced object during a JA behavior exchange (Falck-Ytter et al., 2012; Navab et al., 2012). For that purpose, we created an eye-tracking task to measure RJA in response to a gaze shift with different emotional intensity in ASD and TD preschoolers. In each condition, an actor shifted her gaze through a referenced object with a neutral, mild or strong expression of surprise on her face. A pointing condition also was added, in which the actor indicated the referenced objected with her index and a neutral expression. We expected to observe a reduction in overall time spent on communicative cues (the face in the expressive conditions and the hand in the pointing condition) and a decrease in RJA in the ASD group compared to their TD peers. Second, we hypothesized that the intensity of the emotion expressed by the actor would enhance attention to faces within both the ASD and TD groups, and increase RJA in the ASD group. Third, we predicted that these eye-tracking variables would correlate with adaptive 
behavior, especially in the Communication domain, as measured by the Vineland Adaptive Behavioral Scale, 2nd Edition (Sparrow et al., 2005).

\section{Methods}

\section{Participants}

(Table 1)

\section{ASD participants}

Thirty toddlers and preschoolers (27 males) with ASD aged 19 to 55 months were enrolled in the study. After controlling for data quality, 5 children were excluded from the data analyses (see below for precise exclusion criteria). The final sample size thus consisted of 25 young children (23 males) with ASD aged 19 to 55 months. Demographic information is available in Table 1. Participants with ASD were recruited through French-speaking parent associations and local autism clinics. The Institutional Review Board of the University of Geneva approved the study and parents gave their informed consent before inclusion in the study.

All participants with ASD were evaluated with either the Autism Diagnosis Observation Schedule - Generic (Lord et al., 2000) or the toddler module of the Autism Diagnosis Observation Schedule, second Edition (ADOS-2, Luyster et al. 2009). Fifteen participants were evaluated with Module 1 or 2 of the ADOS-G (Module 1 or 2). Their ADOS-G scores were then transformed into severity scores according to previously published algorithms (Gotham, Pickles, \& Lord, 2008; Gotham, Risi, Pickles, \& Lord, 2006) to allow for comparison between different modules. Using the Gotham et al. transformation (Gotham et al., 2006; 2008), 4 children met criteria for ASD and 11 for Autism. Their mean severity score was $6.46($ S.D. $=1.41)$ out of 10 . Ten other children were evaluated using the toddler module of the ADOS-2 for children younger than 30 months, and 9 of them met criteria for the Moderate to Severe range of concern and 1 for Mild to Moderate range of concern. ADOS assessments were administered and scored by psychologists who met requirements for research reliability. Before inclusion in our study, participants older than 30 months had already received a clinical diagnosis of ASD. For the participants younger than 30 months, high concern for ASD was previously established by a specialized clinical center using the toddler module of the ADOS-2.

\section{TD participants}

Twenty-five TD children (19 males) 14 to 57 months of age participated in the study. After controlling for data quality, 21 TD children (17 males), aged between 14 and 57 months and matched for age with the ASD group were retained for data analyses (see Table 1 for demographics information). 
TD participants were recruited through announcements in the Geneva community. Before participation, they were screened for neurological/psychiatric problems and learning disabilities using a telephone interview and a medical and developmental history questionnaire. Any developmental delay or family history of ASD (including extended family) was grounds for exclusion from the study. Parents gave full informed consent before their children's inclusion in the study. All TD participants were assessed using the ADOS-G. Their mean severity score was 1.04 (S.D.=0.19), and there was no clinical concern for any of them.

\section{Assessment instruments}

\section{Response to joint attention eye-tracking task}

The JA eye-tracking task (JA-task) was designed and created for the study. It consisted of eight videos in which an actor's facial expression varied in intensity in response to a moving object. In all of the videos, the actor is standing behind a table with two identical objects (see Fig 1a). Each video lasts 10 seconds and the timing of the actor looking at the object is identical in all of the videos. The actor starts by looking directly at the camera. After 3 seconds, when the objects on the table begin to move, she orients her attention to one of them with an expression of surprise or with a neutral expression that lasts 6 seconds. During the last second, she shifts to a direct gaze, looking at the camera. There are two videos for each of four conditions. In three of the conditions, the intensity of the actor's expression is either mildly or intensely surprised or neutral. In the fourth condition (pointing), the actor points to the object with a neutral expression on her face while looking at the object. The side (left or right) of the actor's facial orientation and pointing, as well as the objects presented (toy truck, ball, plush rabbit, and flowers), were counterbalanced and the order of the eight videos was randomized within participants. Before each video presentation, a turning wheel on a black background was used to center the participant's attention to the level of the actor's face.

The task was administered using Tobii Studio software (www.tobii.com) on a TX300 Tobii eye-tracker. The sampling rate was $300 \mathrm{~Hz}$ and the video resolution 1920x1080 pixels. The children sat either on their parent's lap or alone on a chair at a distance of approximately $60 \mathrm{~cm}$ from the screen, Tobii's recommendation for minimizing the impact of head movement on gaze data. Before administering the task, all participants completed a five-point calibration procedure designed for toddlers and preschoolers. The procedure consisted of tracking an image of a pet animal to five different points on the screen. Calibration was repeated until each participant hit all five points with both eyes. For analyzing collected data, we used Tobii Studio, version 3.1.6.. When a participant was focused for at least 100ms within a 30-pixel circle, it was considered a fixation point. Following data collection, Area of Interests (AOIs)

(Fig. 1b) delineated the face, the object of interest and the hand in the pointing condition. Visual explorations were operationalized as follows: 1) Time spent on the AOIs (face, objects, hand) was divided by the total time spent on the 
screen for each stimulus, to obtain the percentage of time spent on each AOI; 2) RJA was calculated as the number of correct gaze shifts between the face (or the hand in the pointing condition) and the AOI of the object the actor was looking at (one point per condition when present). Also, we calculated RJA errors as the number of gaze shifts between the face/hand and the AOI of the unreferenced object. A gaze shift was considered successful when the child's gaze went directly from the face/hand to the object within $500 \mathrm{~ms}$ at least one time per video. Thus, each child's score for successful and unsuccessful RJA events ranged from 0 and 8, and from 0 to 2 for each condition (neutral, mild, intense, pointing). 4) Finally, we calculated the time needed to correctly answer to RJA from the moment when the communicative cue (face or hand) was gazing or pointing at the referenced object. For this purpose, we measured the time interval between the exact moment when the actor began orienting herself to the referenced object and the moment when the participant's gaze hit the referenced AOI.

Only data from participants who looked at the screen at least $50 \%$ of the time for each stimulus were included in this study.

(Figure 1)

\section{Behavioral measures of joint attention and communication}

To measure joint attention behaviors, we used the Early Social Communication Scale (ESCS, Mundy, Delgado, Block, \& Venezia, 2003). This play-based instrument allowed us to quantify successful RJA during 14 examiner-prompted opportunities. The ESCS also calculates IJA events initiated by the child. The sessions are video recorded, and the IJA and RJA behaviors are subsequently coded according to a manual. Each video was double-coded and discussed until the raters reached consensus. In our sample, 8 out of 25 participants with ASD did not complete the entire 20-minute ESCS because they disengaged at some point during the evaluation. Given that ESCS scoring is based on the frequency of behaviors across the whole task, we decided to exclude these 8 participants from our ESCS analyses. In the group of TD children, we had to exclude data from 2 out of 21 children for the same reason.

Communication was measured using the Vineland Adaptive Behavior Scales, $2^{\text {nd }}$ edition (VABS-II; Sparrow, 2005). The VABS-II is a parent report that provides standardized scores for adaptive behavior in the Communication, Daily Living Skills, Socialization and Motor skills domains, which contribute to a total Adaptive Behavior score. We successfully collected VABS-II data for all participants. 


\section{Statistical analyses}

To compare the TD and ASD groups on time spent on AOIs (face, hand, objects), RJA behaviors and the timing of RJA behaviors, we either conducted non-parametric Mann-Whitney tests (when the distribution of our dependent variables was not normal) or t-tests (when the distribution was normal) with diagnosis as a fixed variable.

We then used Friedman and Dunn's multiple comparisons non-parametric analyses to explore differences in the four conditions presented in the JA-task (neutral, mild, intense and point) for each group. Next, we used correlation analysis (Spearman) to test for a relationship between RJA on the eye-tracking JA-task, RJA on the ESCS and measures from the Vineland-II. In general, the results were considered significant at $p \leq 0.05$. We used Bonferroni corrections for multiple comparisons $(p \leq 0.0125)$ when we applied multiple intra-group comparisons. This concerns the correlations between the RJA measures and the VABS-II for the ASD group (which had four domain scores). All tests were run using GraphPad Prism 6.0 for Mac OSX. Normality was tested using Agostino-Pearson omnibus normality test.

\section{Results}

\section{Eye-tracking Joint Attention Task}

\section{Time spent on communicative cues}

Given that ASD participants spent less time overall looking at the screen compared to their TD peers ( $U=95$, $p=0.001$, see Table 2), we calculated a percentage of time spent on each AOI, by dividing time spent on each AOI divided by total time spent on the screen during each video.

(Table 2)

Across the eight videos, the ASD group spent less of their time on the actor's face compared to the TD group (See Fig. 2.a. and Table 2). When exploring the four conditions separately, time spent on faces was reduced in the ASD group compared to the TD group for the neutral, mild and intense conditions, but not the point condition (see Table 2). Also, we did not observe a group difference in the percentage of time spent on the hand in the pointing condition (see Table 2). We did not find differences between ASD and TD children on time spent looking at either the referenced or unreferenced objects across the eight trials or for each condition separately (see Table 2).

Using Friedman analysis, we then examined the percentage of time spent on the actor's face within each group by condition (neutral, mild, intense, pointing). In the ASD group, we did not observe any difference in the amount of time spent on the face between the four conditions $\left(F_{r}=8.85, p=0.21\right.$, see Fig. 2.b. $)$. There was, however, a significant difference for the time spent on the face between the four conditions within the TD group $\left(F_{r}=171.2, p<0.001\right.$, see Fig. 
2.b.). Using Dunn's multiple comparisons test we observed that time spent on faces in the TD group for the intense condition was greater than the time spent on faces in the other three conditions: intense-neutral comparison $(p=0.04)$, intense-mild comparison $(p=0.002)$, intense-point comparison $(\mathrm{p}=0.003)$. We did not observe a significant relationship between the time spent on the face and the age of the ASD $\left(r_{s}=-0.14, p=0.51\right)$ or $\operatorname{TD}\left(r_{s}=0.30, p=0.19\right)$ participants.

(Figure 2)

\section{Responses to joint attention (RJA)}

Children with ASD responded to fewer of the RJA opportunities compared to their TD peers ( $U=74$, $p<0.0001$ ). Out of the eight occasions (one per video) to correctly follow the actor's gaze or pointing, the TD group responded to $67.3 \%($ S.D. $=21.5)$ of the opportunities and children with ASD responded to 35.0\% (S.D.=19.8) (see Fig. 3.a.).

We then compared the groups on the number of participants that correctly followed the actor's gaze shift or pointing (RJA behavior) during the two videos from each condition (see Fig. 3.b.). For the neutral and mild conditions, there was a significant difference between the groups $(U=83.5, \mathrm{p}<0.0001 ; U=121.5, p=0.0005)$, with ASD participants following the actor's gaze less often than their TD peers. A difference in RJA between the two groups was also observed in the pointing condition $(U=147, p=0.005)$. No group difference was found in the RJA intense condition $(U=206.5, p=0.19)$. We did not observe a relationship between the percentage of correct gaze shifts from face to the object referenced from the actor (RJA) and age of the participants in the ASD $\left(r_{s}=0.26, \mathrm{p}=0.21\right)$ or $\mathrm{TD}\left(r_{s}=0.30, p=0.19\right)$ groups. We subsequently performed a Friedman test. We observed a significant within-group effect in the TD group for RJA behavior during the four conditions $\left(F_{r}=10.60, p=0.01\right)$. Dunn's test for multiple comparisons within the TD group was not significant. However, there is evidence for a trend toward significance between the mild and the pointing conditions (with an increased rate of RJA in the pointing condition compared to the mild condition), $(p=0.10)$. No other significant differences were observed among the comparisons of the other conditions in the TD group (p>0.56). Within the ASD group, the Friedman test was significant for RJA across the four conditions $\left(F_{r}=31.40, p<0.001\right)$. Dunn's test for multiple comparisons subsequently showed significant differences on RJA between the neutral and intense conditions $(p=0.007)$ and the neutral and pointing conditions $(p=0.006)$, with RJA shifts less frequent during the neutral condition. Dunn's test for multiple comparisons also demonstrated significant differences on RJA between the mild and the intense $(p=0.004)$ conditions and the mild and the pointing conditions $(p=0.003)$, with RJA events less frequent during the mild condition.

Children with ASD and TD children did not differ on the number of incorrect gaze shifts from communicative cue to the unreferenced object (RJA errors) $(U=185, p=0.08)$. We did, however, observe a trend toward significance, with the ASD group showing more overall errors than the TD group (ASD group, 19.0\%, S.D.=14.9; TD group 11.3\%, 
S.D.=11.8). No difference was observed between the ASD and the TD groups for the separate conditions, though we observed a trend toward significance in the point and the neutral condition (neutral, $U=203.5, p=0.12 ;$ mild, $U=245$, $p=0.64$; intense, $U=247.5, p=0.63$; point, $U=201, p=0.08$ ).

We subsequently compared participants' correct and incorrect gaze shifts to the referenced and unreferenced objects to address whether correct instances of RJA could be due to chance. To this end, we compared the percentage of correct RJA (correct gaze shifts from the communicative cue to the referenced object) with the percentage of incorrect gaze shifts from the communicative cue to the unreferenced object. Our results show that in both ASD and TD groups, the percentage of gaze shifts to the referenced objects was higher than the percentage of gaze shifts to the unreferenced objects (TD group, $\mathrm{W}=231, \mathrm{p}<0.0001$; ASD group, $\mathrm{W}=144, \mathrm{p}=0.0008$ ).

Finally, our analyses of the timing of RJA behaviors between the TD and the ASD groups did not reveal differences in the time it took to hit the referenced object across all trials (ASD: 2.3 sec., S.D.=0.6; TD: 2.4 sec., S.D. $=0.9 ; \mathrm{t}=0.39, \mathrm{p}=0.70$ ) or when analyzing each emotion separately (all $\mathrm{p}>0.53$ ).

(Figure 3)

\section{Relationship between eye-tracking and behavioral measures for RJA at the ESCS (Mundy et al., 2003)}

The group of children with ASD showed 60.7\% (S.D.=39.6) of correct RJA on the ESCS, which was statistically different from the percentage of correct RJA demonstrated by the TD group at the same evaluation $(92.18 \%$, S.D. $=17.79 ; U=79, p=0.01)$. Within the ASD group, RJA on our eye-tracking JA-task were correlated with behavioral RJA on the ESCS $\left(r_{s}=0.62, p=0.009\right.$, see Fig. 4). Within the TD group, we did however not observe a relationship between responding to RJA on the JA-task measure and behavioral RJA on the $\operatorname{ESCS}\left(r_{s}=0.28, p=0.31\right)$.

(Figure 4)

\section{RJA and adaptive behavior}

As shown in Table 1, the ASD group had reduced communication skills on the VABS-II compared to their TD peers as well as lower adaptive behavior on the other adaptive behavior scores: Socialization, Daily Living Skills, Motor Skills and Total Adaptive Behavior. Within the ASD group, we did not observe correlations between Communication on the VABS-II and RJA on the ESCS $\left(r_{s}=0.39, p=0.12\right)$, where we found a weak trend toward significance, or on the JA-task $\left(r_{s}=0.20, p=0.34\right)$ in the ASD group. Nor were relationships observed between RJA measures on the ESCS and Daily Living Skills $\left(r_{s}=-0.16, p=0.54\right)$, Socialization $\left(r_{s}=0.33, p=0.20\right)$ or Motor Skills $\left(r_{s}=-\right.$ 0.06, $p=0.78$ ) in ASD participants. Nor did we observe a significant relationship between JA-task and Daily Living 
Skills $\left(r_{s}=-0.02, p=0.90\right)$, Socialization $\left(r_{s}=0.22, p=0.29\right)$ or Motor skills $\left(r_{s}=0.10, p=0.62\right)$. We also did not observe correlations between RJA scores on the JA-task and the four sub-scales of the VABS-II (Communication, $r_{s}=0.27$, $p=0.45$; Daily Living Skills, $r_{s}=0.03, p=0.89$; Socialization, $r_{s}=0.16, p=0.57$; Motor Skills, $\left.r_{s}=-0.03, p=0.92\right)$ in the TD group. Nor were significant correlations observed between RJA scores on the ESCS and the VABS-II subscales in TD participants (Communication, $r_{s}=0.31, p=0.27$; Daily Living Skills, $r_{s}=0.14, p=0.61$; Socialization, $r_{s}=0.33, p=0.24$; Motor Skills, $\left.r_{s}=0.13, p=0.66\right)$.

\section{Discussion}

In the current study, we report reduced time spent on faces in preschoolers with autism spectrum disorders (ASD) in a joint attention eye-tracking task (JA-task). As expected, our sample of young children with ASD showed greater difficulty responding to joint attention (RJA) compared to typically developing (TD) peers. Furthermore, our results show that intense emotional expression increased time spent on the actor's face in TD participants, but not in ASD participants. However, ASD participants did follow a greater number of RJA opportunities during the intense surprise and finger pointing conditions when compared to the neutral expression and mild surprise conditions. Further, children on the autism spectrum did not differ from their TD peers on the number of correct gaze shifts during the intense surprise condition but differed from TD children during the other three conditions. Finally, within the ASD group, RJA on the JA-task was positively correlated with RJA on a behavioral task (the Early Social Communication Scale, ESCS, Mundy et al. 2003). We did not, however, observe a correlation between either eye-tracking or behavioral measures of RJA and communication measures on a parent report (the Vineland Adaptive Behavioral Scale, 2nd edition, VABS-II, Sparrow et al., 2005). It is worth considering why RJA during the eye-tracking task, RJA behavioral measurements, and further communication scores were not significantly correlated in the TD group. One reason may be the existence of a ceiling effect in the TD group for RJA behavioral measurements on the ESCS. TD participants demonstrated perfect RJA on the ESCS, with a group average of 92.18\% (S.D.=17.79).

A reduction in time spent on the actor's face during the JA-task in children with autism compared to TD group corroborates previous findings from Chawarska et al. (2012). Our results shed light on the complex relationship between faces and RJA behavior. Reduced orienting towards faces in infants with ASD may hinder the development of dyadic interactions, which are essential to the construction of shared attention behaviors like JA (Jones, Carr, \& Klin, 2008; Osterling et al., 2002; Shic, Bradshaw, Klin, Scassellati, \& Chawarska, 2011) and indispensable to learning about the environment. Previously published joint attention eye-tracking paradigms have explored both gazing at faces (time spent on faces), as well as transitions from faces to a referenced object. In our study, we first analyzed the time TD and ASD preschoolers spent gazing at faces. Within the TD sample, the time spent on the actor's face increased during JA 
inducing-videos when the actor expressed intense surprise. This result is consistent with studies of healthy adults (for a review see Vuilleumier, 2002) showing that emotional expressions heighten attention to faces when compared to neutral expressions, a phenomenon that has additionally been observed during a specific gaze-directed paradigm (Jones, DeBruine, Little, Conway \& Feinberg, 2006). In contrast to the TD group, time spent on the actor's face did not vary significantly between conditions in our ASD participants. Second, we analyzed gaze shift transitions from the face to the referenced object, a variable that we examined independently from the time spent on the actor's face. Similar to previous studies, we report a reduction in RJA gaze shift in young children with ASD in behavioral and eye-tracking settings (Dawson et al., 2004; Falck-Ytter et al., 2012; Mundy et al., 1986; Navab et al., 2012). However, we here show for the first time that children with ASD improve their gaze shift skills in the intense and the pointing conditions, emphasizing the role that emotional expression and pointing play in RJA. Although the time the TD group spent on the face increased during the intense surprise condition, they did not demonstrate increased RJA transitions from face to the referenced object. Children with ASD, however, showed increased RJA transitions from the face to the referenced object during the intense condition despite not spending more time gazing at the intense emotional face. This implies that the enhanced time spent on the face behaves separately from the number of RJA gaze shifts. Accordingly, to the best of our knowledge, a direct association between the time spent on faces and improved RJA gaze shifts has never been shown. Our results support the idea that children with ASD perform better in the intense and point conditions because the content of the facial expression/gesture makes the gaze shift clearer and easier to follow. Carpenter et al. (2002) previously showed that preschoolers with ASD had trouble following gaze behavior during a JA opportunity, but that the participants were helped when the gaze shift was coupled with a pointing gesture. It is important to mention that the actor's movements were more pronounced in the intense and pointing conditions compared to the neutral and mild conditions. In the group of children with ASD, the time spent on communicative cues was not statistically different between conditions. It is yet possible that the movement in the intense and point conditions made the cues more salient, helping to explain increased RJA in children with ASD during these two conditions. Future work should include additional control conditions (e.g. comparing static and dynamic conditions) to explore the effect of movement associated with communicative cues on gaze shifting. Other studies in healthy adults show the benefits of emotional expression, whereby fearful faces reduce response time to RJA (Putman, Hermans, \& van Honk, 2006; Tipples, 2010) and emotion (fear, surprise or anger) can enhance gaze shift through a referenced cue (Lassalle et al. 2015). However, a study by Uono et al. (2009) showed that fearful facial expressions enhanced gaze shift reaction time in a group of adolescents with typical development, but not in a group of adolescents with Asperger's syndrome. The authors suggest that integration of emotion in RJA may be impaired in individuals on the autism spectrum. By contrast, our results support a facilitative role of emotional expression (surprise) for gaze shifts in young children with autism. There are several explanations for the discrepancy between our results and Uono et al's findings (2009): a difference in the 
variables used to measure RJA gaze shifts (reaction time vs. occurrences), different emotional expressions (fearful vs. surprise) and differences in age and/or developmental abilities between the group of participants involved in the two studies (adolescents with Asperger syndrome vs. preschoolers with ASD). Future work should address these methodological differences to further understand the alleged role of emotional expressions in RJA throughout the development of individuals with ASD.

Our study lends substantial empirical support to clinical guidelines that suggest that intense facial expressions can clarify the communicative content of social referencing behaviors (Ingersoll \& Dvortcsak, 2006; Taylor \& Hoch, 2008; Mead \& Mataric, 2010; Rogers \& Dawson, 2010). Our data also imply that the positive effect of emotional expression on social referencing (Mumenthaler and Sander, 2015; Bayliss et al. 2006; 2007) may be preserved in young children with ASD who might also be benefitting of emotional facial expression to improve their gaze shift skills. On these bases, we recommend that the positive effect of intense emotional expression on social referencing and affective sharing in preschoolers with ASD, as well as in typically developing preschoolers, can be adapted to help individuals with ASD learn to follow affective cues.

Finally, we found a positive correlation in ASD participants between eye-tracking and behavioral measures of RJA, the ESCS (Mundy et al. 2003). An eye-tracking measure for RJA was similarly related to a behavioral measure for RJA in a group of 18-month-old children with ASD in one of our previous studies (Navab et al., 2012), provided further support for eye-tracking as a valid and standardized way of observing gaze shift responses in young children on the autism spectrum. By contrast, we did not see a relationship between RJA (on the JA-task and the ESCS) and Communication on the VABS-II. The relationship between behavioral RJA and communication skills in the group of children with ASD yet showed a weak trend toward significance. One explanation for this may be the young age of our participants. Mundy and collaborators (Mundy et al., 1990; Mundy \& Gomes, 1998) have suggested that the relationship between JA skills and language development may be moderated by the developmental level of the children, making RJA easier to track later in life when language development is further along. Given the importance of RJA skills for the language development, it appears to be crucial to detect RJA abnormalities as soon as possible to intervene on gaze shift responses and thus support preverbal and subsequent verbal communication skills, which both represent core difficulties of ASD.

\section{Limitations}

The present study has several limitations. First, we used few items per condition in our JA-task (2 per condition) to ensure that our young participants were able to pay attention to the task from beginning to end. This was important to avoid an ascertainment bias in our sample and include a range of participants and not only those with longer attention spans. Second, the two groups were matched on chronological age, but not on developmental abilities, which may have 
accentuated group differences on the dependent variables of interest. Future studies examining the effect of emotional expression on RJA should strive to compare ASD children with a developmentally age-matched group when possible. Third, our relatively small sample size may have reduced our statistical power and ability to detect differences. This point specifically concerns the following analyses: 1 . The trend toward increased RJA in the TD group during the pointing compared to the mild condition $(p=0.10)$, which would indicate that similar to that of ASD children, TD children's RJA varies by condition. 2. Children with ASD tended toward less accurate RJA (i.e. looking at the unreferenced object) during the eye-tracking task compared to TD participants. This trend appeared to be specific to the pointing $(p=0.08)$ and the neutral $(p=0.12)$ conditions, suggesting that replicating the study in a bigger sample size would reveal accuracy differences in RJA between TD and ASD children. 3. Within ASD participants, a trend toward a significant correlation was observed between RJA scores on the eye-tracking task and Communication scores from the VABS-II $(p=0.12)$. This echoes previous studies that report a relationship between JA and communication abilities (Murray et al., 2008, Whalen \& Schreibman, 2006; Jones, Carr \& Feeley, 2006). Finally, the correlation analyses between the RJA behavioral measurements (using the ESCS) and the VABS-II also were underpowered due to a large number of dropouts from the ESCS evaluation. Taken together, especially in light of clinical observations about the role that emotional intensity plays in RJA in young children with ASD, these preliminary results encourage further exploration of the effect of emotional intensity on RJA in young TD and ASD children.

\section{Implications}

Eye-tracking represents a unique tool for quantifying joint attention responses (RJA) in young children with ASD. In this study, we provide empirical evidence showing that more intense emotional expressions can facilitate gaze shifting in the early development of RJA skills, an idea that was previously supported only by clinical guidelines. The results from this study can inform knowledge about attention to emotional expressions in autism and improve our understanding of how early interventions may work to increase preverbal experiences in young children with ASD.

\section{Acknowledgments}

The authors would like to thank all the families who kindly volunteered to participate, as well as Stéphanie Baudoux, Léa Chambaz, Sophie Diakonoff, Nada Kojovic, Matthieu Mansion, Isaline Mottet and Myriam Speller for their help with data collection. This study was supported by the National Center of Competence in Research "Synapsy," financed by the Swiss National Science Foundation (SNF, Grant number: 51AU40_125759) and by the "Fondation Pôle Autisme" (http://www.pole-autisme.ch). Marie Schaer is further supported by an individual fellowship from the SNF (\#158831). 


\section{References}

Adamson, L.B., Deckner, D.F. \& Bakeman, R. (2010). Early interests and joint engagement in typical development, autism, and down syndrome. J Autism Dev Disord, 40, p. 665-676.

Aubineau, L.-H., Le Driant, B., \& Vandromme, L. (2015). L'attention conjointe, 40 ans dévaluations et de recherches de modélisations. L'Année Psychologique, 115(1), p. 141-174.

Bayliss, A. P., Frischen, A., Fenske, M. J., \& Tipper, S. P. (2007). Affective evaluations of objects are influenced by observed gaze direction and emotional expression. Cognition, 104(3), 644-653.

Bayliss, A. P., Paul, M. A., Cannon, P. R., \& Tipper, S. P. (2006). Gaze cuing and affective judgments of objects: I like what you look at. Psychonomic Bulletin \& Review, 13(6), 1061-1066.

Carpenter, M., Pennington, B. F., \& Rogers, S. J. (2002). Interrelations Among Social-Cognitive Skills in Young Children with Autism. Journal of Autism and Developmental Disorders, 32(2), 91-106.

Charman, T. (2003). Why is joint attention a pivotal skill in autism? Philosophical Transactions of the Royal Society of London. Biological Sciences, 358(1430), 315-324.

Charman, T., Baron-Cohen, S., Swettenham, J., Baird, G., Drew, A., \& Cox, A. (2003). Predicting language outcome in infants with autism and pervasive developmental disorder. International Journal of Language \& Communication Disorders, 38(3), 265-285.

Chawarska, K., Klin, A., \& Volkmar, F. (2003). Automatic attention cueing through eye movement in 2-year-old children with autism. Child Development, 74(4), 1108-1122.

Chawarska, K., Macari, S., \& Shic, F. (2012). Context modulates attention to social scenes in toddlers with autism. Journal of Child Psychology and Psychiatry, 53(8), 903-913.

Chevallier, C., Kohls, G., Troiani, V., Brodkin, E. S., \& Schultz, R. T. (2012). The social motivation theory of autism. Trends in Cognitive Sciences, 16(4), 231-239.

Dawson, G., Bernier, R., \& Ring, R. H. (2012). Social attention: a possible early indicator of efficacy in autism clinical trials. J Neurodev Disord.

Dawson, G., Toth, K., Abbott, R., Osterling, J., Munson, J., Estes, A., \& Liaw, J. (2004). Early social attention impairments in autism: Social orienting, joint attention, and attention to distress. Developmental Psychology, 40(2), 271-283.

Falck-Ytter, T., Fernell, E., Hedvall, A. L., Hofsten, von, C., \& Gillberg, C. (2012). Gaze performance in children with autism spectrum disorder when observing communicative actions. Journal of Autism and Developmental Disorders, 42(10), 2236-2245.

Frischen, A., Bayliss, A. P., and Tipper, S. P. (2007). Gaze cueing of attention: visual attention, social cognition, and individual differences. Psychol. Bull. 133, 694-724.

Gotham, K., Pickles, A., \& Lord, C. (2008). Standardizing ADOS scores for a measure of severity in Autism Spectrum Disorders. Journal of Autism and Developmental Disorders, 39(5), 693-705.

Gotham, K., Risi, S., Pickles, A., \& Lord, C. (2006). The Autism Diagnostic Observation Schedule: Revised algorithms for improved diagnostic validity. Journal of Autism and Developmental Disorders, 37(4), 613-627. 
Hoehl, S., Wiese, L., and Striano, T. (2008). Young infants' neural processing of objects is affected by eye gaze direction and emotional expression. PLOS ONE 3:e2389

Hofsten, Claes, Dahlström, E., \& Fredriksson, Y. (2005). 12- month- old infants' perception of attention direction in static video images. Infancy, 8(3), 217-231.

Ingersoll, B., \& Dvortcsak, A. (2006). Teaching Social Communication to Children with Autism. New York, NY: Guilford Press.

Itier, R. J., \& Batty, M. (2009). Neural bases of eye and gaze processing: The core of social cognition. Neuroscience \& Biobehavioral Reviews, 33(6), 843-863.

Jones, B. C., DeBruine, L. M., Little, A. C., Conway, C. A. \& Feinberg, D. R. (2006). Integrating gare direction and expression in preferences for attractive faces. Psychological Science, 17, 588-591.

Jones, E. A., \& Carr, E. G. (2004). Joint attention in children with autism theory and intervention. Focus on Autism and Other Developmental Disabilities, 19(1), 13-26.

Jones, E. A., Carr, E. G., \& Feeley, K. M. (2006). Multiple effects of joint attention intervention for children with autism. Behavior Modification, 30(6), 782-834.

Jones, W., \& Klin, A. (2013). Attention to eyes is present but in decline in 2-6-month-old infants later diagnosed with autism. Nature, 504(7480), 427-431.

Jones, W., Carr, K., \& Klin, A. (2008). Absence of preferential looking to the eyes of approaching adults predicts level of social disability in 2-year-old toddlers with autism spectrum disorder. Archives of General Psychiatry, 65(8), 946-954.

Jones, E.A., Carr, E.G. \& Feeley, K.M. (2006). Multiple effects of joint attention intervention for children with autism. Behav Modif, 30, 782-834.

Lassalle, A., \& Itier, R. J. (2015). Emotional modulation of attention orienting by gaze varies with dynamic cue sequence. Visual Cognition, 23(6), 720-735.

LeComer, L. F. (2009). The socially included child. New York, NY: Berkley Books.

Leekam, S. R., \& Ramsden, C. A. H. (2006). Dyadic orienting and joint attention in preschool children with autism. Journal of Autism and Developmental Disorders, 36(2), 185-197.

Lord, C., Risi, S., Lambrecht, L., Cook, E. H., Leventhal, B. L., DiLavore, P. C., et al. (2000). The autism diagnostic observation schedule-generic: A standard measure of social and communication deficits associated with the spectrum of autism. Journal of Autism and Developmental Disorders, 30(3), 205-223.

Mastrangelo, S. (2009). Harnessing the power of play: Opportunities for children with Autism Spectrum Disorders. Teaching Exceptional Children, 42(1), 34-44.

Mead, R. \& Mataric, M.J. (2010). Automated caricature of robot expressions in socially assistive human-robot interaction. In the the 5th ACM/IEEE international conference on uuman-robot interaction (HRI'09) worshop on what collaborations with the arts have to say about. New York, NY: ACM.

Mumenthaler, C., \& Sander, D. (2012). Social appraisal influences recognition of emotions. Journal of Personality and Social Psychology, 102(6), 1118-1135.

Mumenthaler, C., \& Sander, D. (2015). Automatic integration of social information in emotion recognition. Journal of Experimental Psychology. General, 144(2), 392-399.

Mundy, P., \& Gomes, A. (1998). Individual differences in joint attention skill development in the second year. Infant Behavior and Development, 21(3), 469-482.

Mundy, P., \& Sigman, M. (1989). The theoretical implications of joint-attention deficits in autism. Development and Psychopathology, 1(03), 173-183. 
Mundy, P., Block, J., Delgado, C., Pomares, Y., Van Hecke, A. V., \& Parlade, M. V. (2007). Individual differences and the development of joint attention in infancy. Child Development, 78(3), 938-954.

Mundy, P., Delgado, C., Block, J., \& Venezia, M. (2003). Early social communication scales (ESCS). Florida, FL: Coral Gables.

Mundy, P., Sigman, M., \& Kasari, C. (1990). A longitudinal study of joint attention and language development in autistic children. Journal of Autism and Developmental Disorders, 20(1), 115-128.

Mundy, P., Sigman, M., Ungerer, J., \& Sherman, T. (1986). Defining the social deficits of autism: The contribution of non-verbal communication measures. Journal of Child Psychology and Psychiatry, 27(5), 657-669.

Murray, D.S., Creaghead, N.A., Manning-Courtney, P., Shear, P.K., Bean, J. \& Prendeville, J.A. (2008). The relationship between joint attention and language in children with Autism Spectrum Disorders. Focus Autism Other Dev Disabl, 23, 5-14.

Navab, A., Gillespie Lynch, K., Johnson, S. P., Sigman, M., \& Hutman, T. (2012). Eye-Tracking as a measure of responsiveness to joint attention in infants at risk for autism. Infancy, 17(4), 416-431.

Niedzwiecka, A. \& Tomalski, P (2015). Gaze-cueing effect depends on facial expression of emotion in 9- to 12months-ols infants. Frontiers in psychology, 6, e1-e11.

Osterling, J. A., Dawson, G., \& Munson, J. A. (2002). Early recognition of 1 -year-old infants with autism spectrum disorders versus mental retardation. Development and Psychopathology, 14(02), 239-251.

Putman, P., Hermans, E., \& van Honk, J. (2006). Anxiety meets fear in perception of dynamic expressive gaze. Emotion, 6(1), 94-102.

Rogers, S. J. \& Dawson, G. (2010). Early Start Denver Model for young children with autism: Promoting language, learning and engagement. New York, NY: Guildford Press.

Rogers, S. J., Estes, A., Lord, C., Vismara, L., Winter, J., Fitzpatrick, A., et al. (2012). Effects of a brief Early Start Denver Model (ESDM)-Based parent intervention on toddlers at risk for Autism Spectrum Disorders: A randomized controlled trial. Journal of the American Academy of Child and Adolescent Psychiatry, 51(10), 10521065 .

Shic, F., Bradshaw, J., Klin, A., Scassellati, B., \& Chawarska, K. (2011). Limited activity monitoring in toddlers with autism spectrum disorder. Brain Research, 1380, 246-254.

Soussignan, R., Schaal, B., Boulanger, V., Garcia, S., \& Jiang, T. (2015). Emotional communication in the context of joint attention for food stimuli: Effects on attentional and affective processing. Biological Psychology, 104, 173183.

Sparrow, S. S., Cicchetti, V. D., \& Balla, A. D. (2005). Vineland adaptive behavior scales (2nd ed.). MN: American

Taylor, B. A., \& Hoch, H. (2008). Teaching children with autism to respond to and initiate bids for joint attention. Journal of Applied Behavior Analysis, 41(3), 377-391.

Teufel, C., Gutmann, A., Pirow, R., \& Fischer, J. (2010). Facial expressions modulate the ontogenetic trajectory of gaze-following among monkeys. Developmental Science, 13(6), 913-922.

Tipples, J. (2010). Fear and fearfulness potentiate automatic orienting to eye gaze. Cognition \& Emotion, 20(2), 309320. Journal of experimental child psychology.

Toth, K., Munson, J., Meltzoff, A. N., \& Dawson, G. (2006). Early predictors of communication development in young children with Autism Spectrum Disorder: Joint attention, imitation, and toy Play. Journal of Autism and Developmental Disorders, 36(8), 993-1005. 
Uono, S., Sato, W., \& Toichi, M. (2009). Dynamic fearful gaze does not enhance attention orienting in individuals with Asperger's disorder. Brain and cognition, 71, 229-233.

Vuilleumier, p. (2002). Facial expression and selective attention. Current opinion in psychiatry, 15, 291-300.

Whalen, C. \& Schreibman, L. (2006). The Collateral effects of joint attention training on social initiations, positive affect, imitation, and spontaneous speech for young children with autism. Journal of autism and developmental disorders, 36, 655-664. 
Figure 2. Screenshot examples of our JA-task. a. Examples for the mild, intense and point condition. In the pointing condition, the hand was also considered as an AOI. b. Drawing representation of AOI's on the face (in orange) and on the looked object (in blue).

Figure 2.a. Percentage of time spent on faces when considering the entire task (8 stimuli) in ASD and TD participants. b. Percentage of time spent on faces in TD and ASD group within each condition (neutral, mild, intense, point). Asterisks represent statistically significant difference within the TD group between the intense and the neutral, mild and point conditions $(\mathrm{p}<0.05)$.

Figure 3.a. Percentage of correct answers to RJA in our JA eye-tracking task in all conditions merged and within ASD and TD groups. $b$. Number of participants that correctly answered at RJA among each condition (neutral, mild, intense, point). Each condition was presented twice and the graphs represent the number of success on 2 attempts. The horizontal dotted line, represent the mean $(\mathrm{m})$ of correct bids for each condition and for each group.

Figure 4. Correlation between the ratio of correct RJA in our eye-tracking JA-task and the ratio of correct behavioral RJA at the ESCS. 
a.

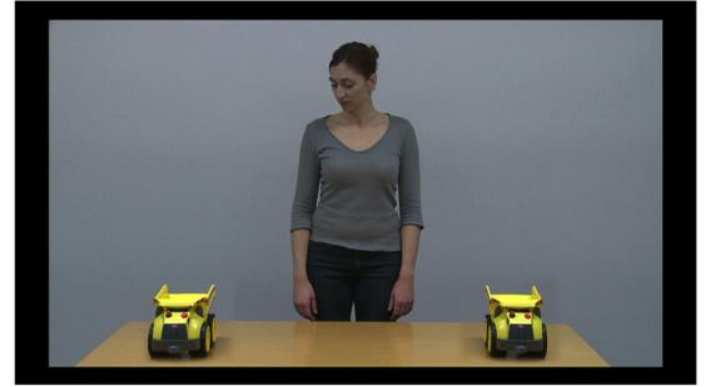

Neutral

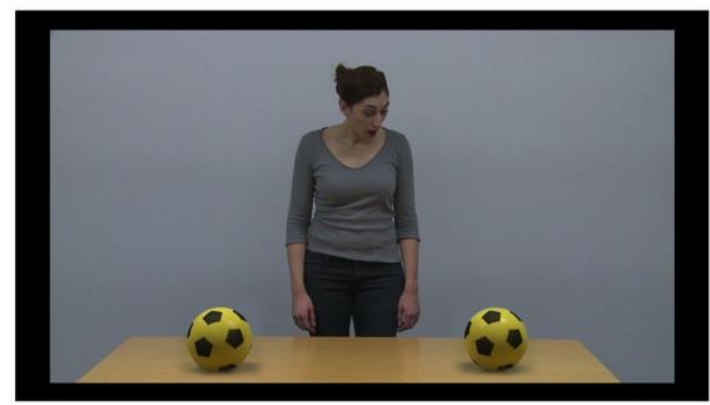

Intense

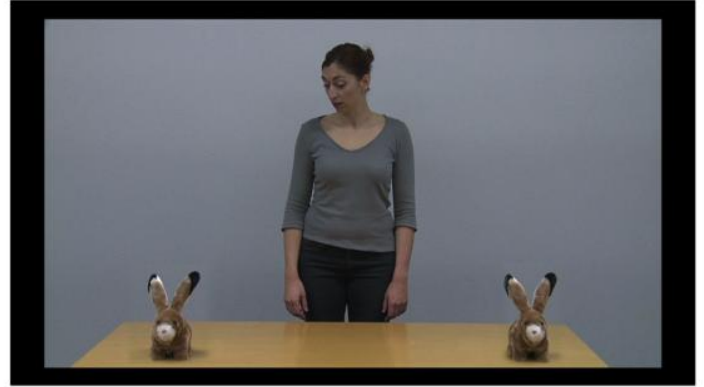

Mild

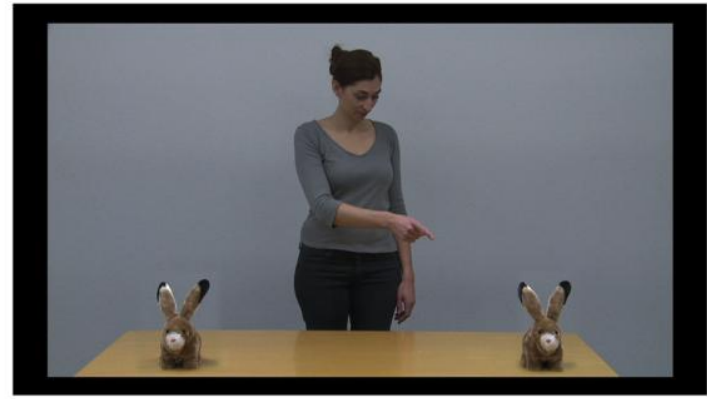

Point

b.

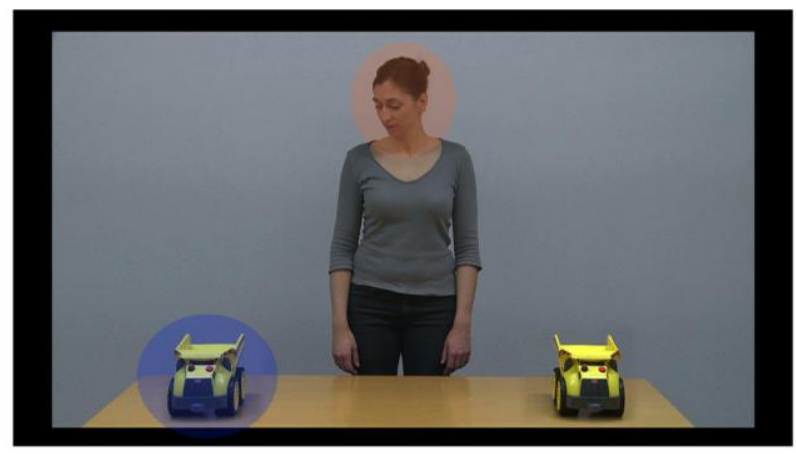


a.

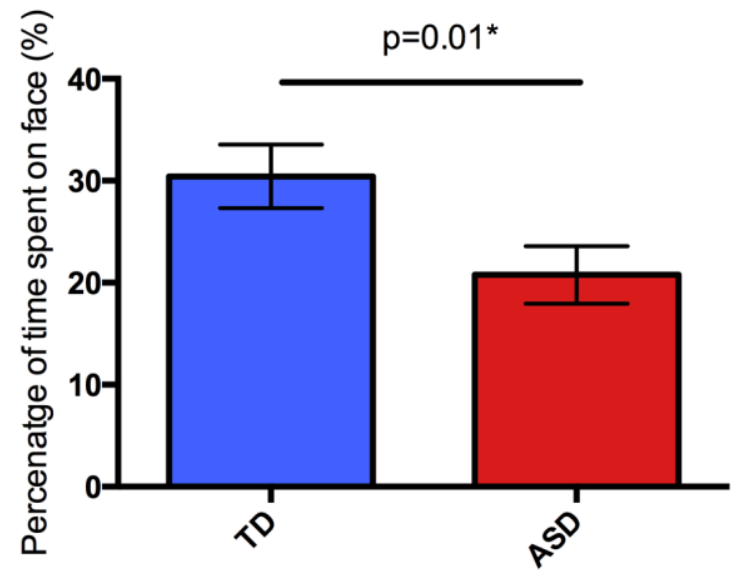

b.
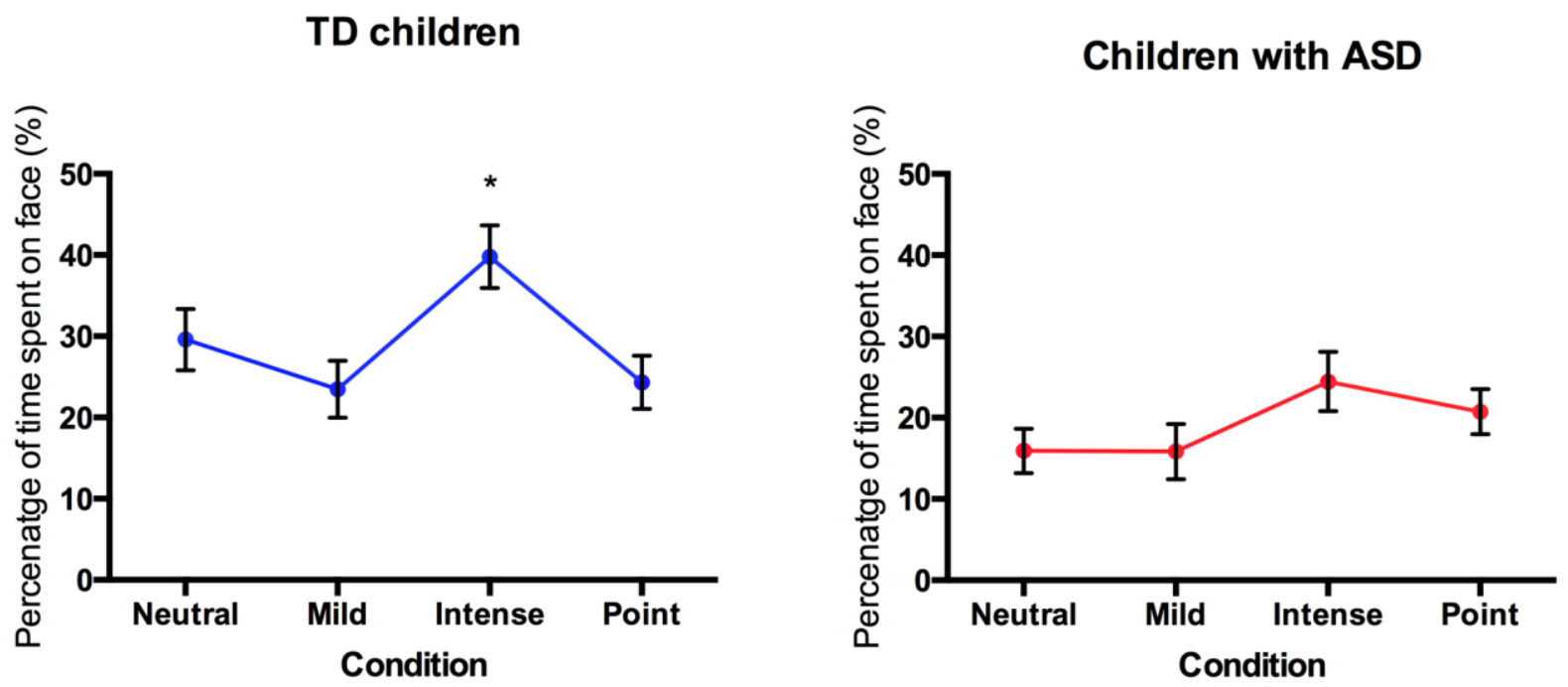
a.

b.

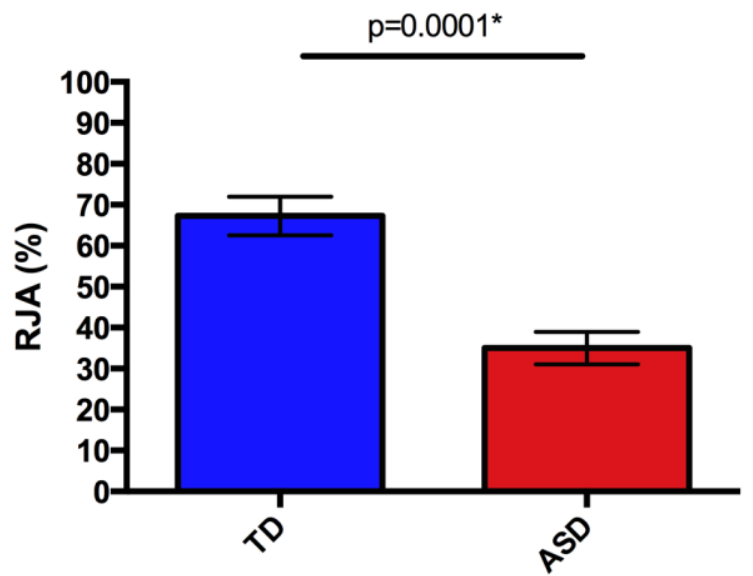

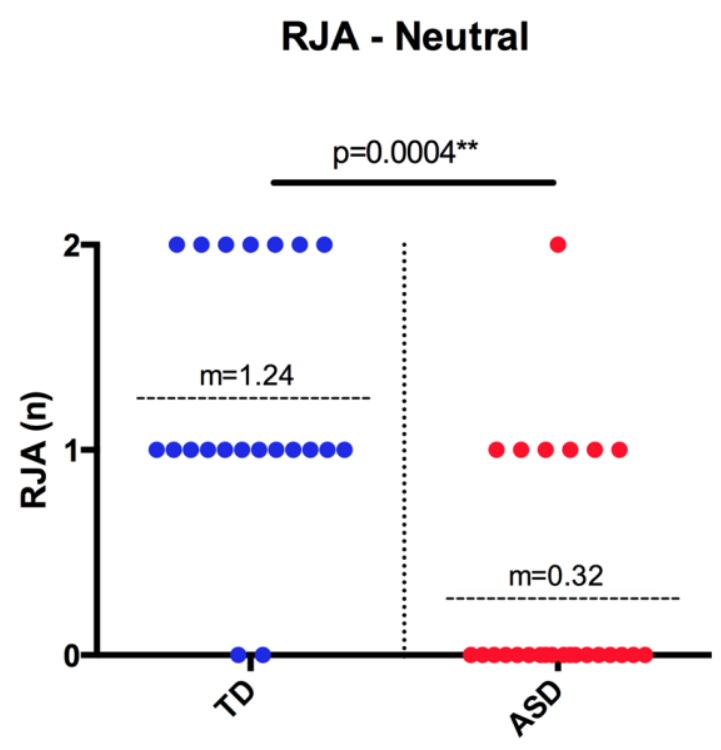

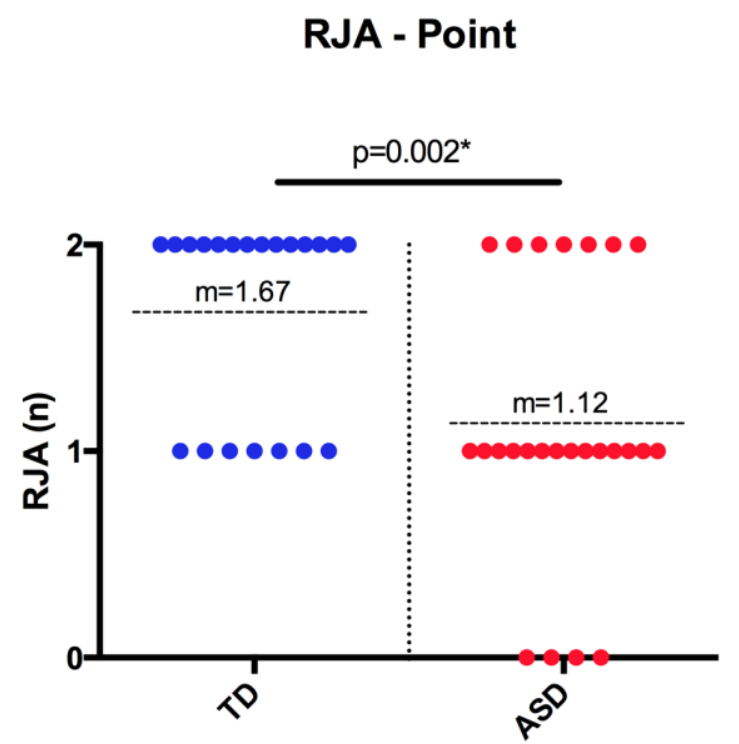

RJA - Mild

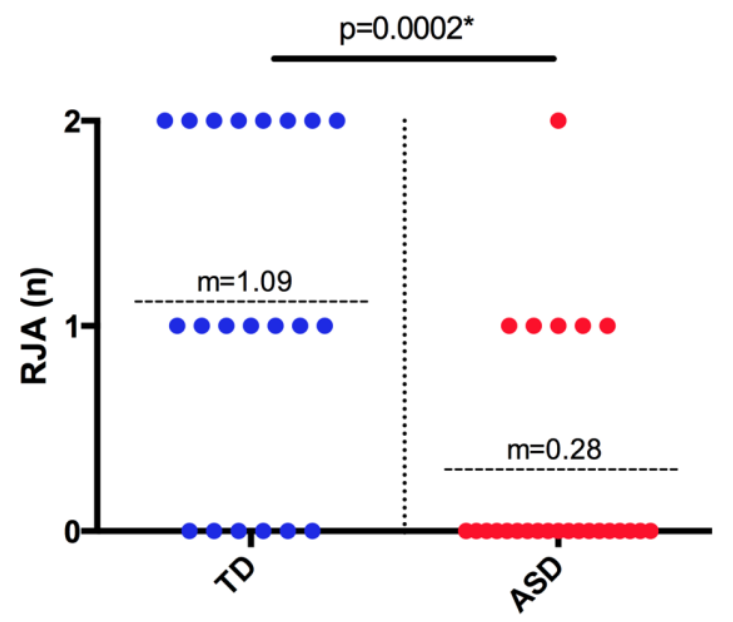

RJA - Intense

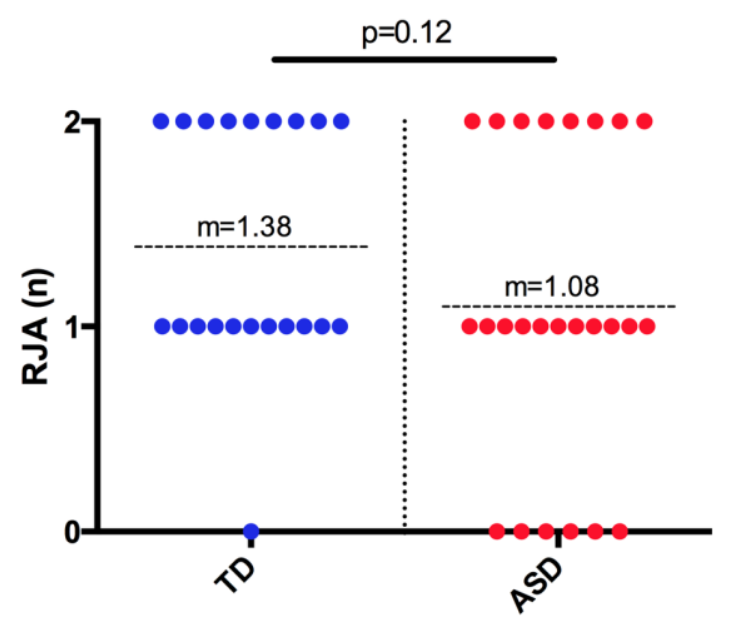




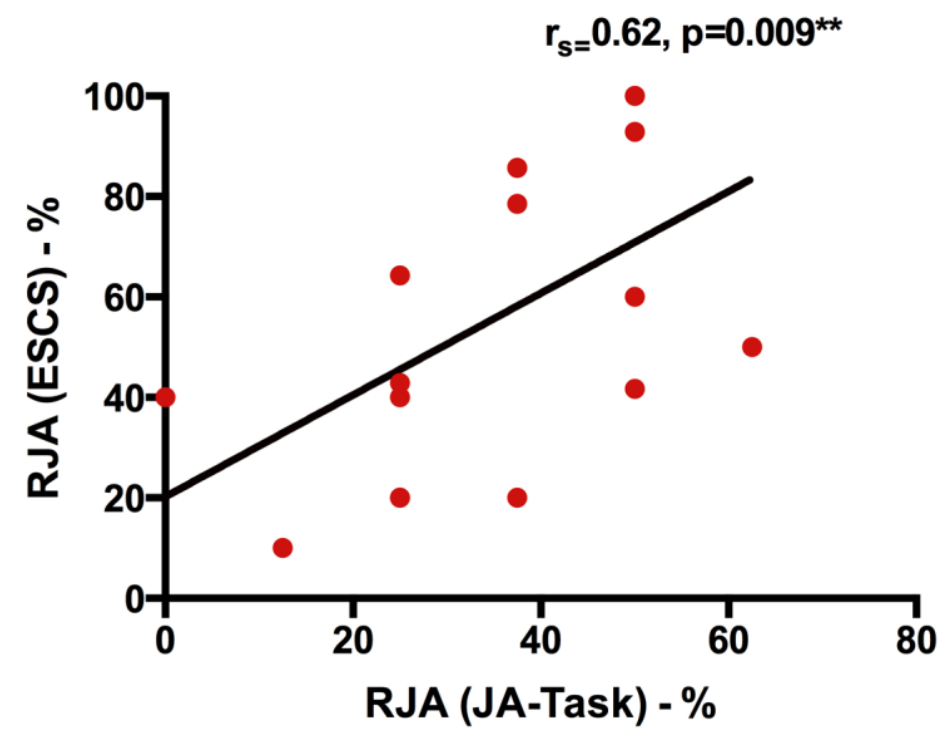




\begin{tabular}{|c|c|c|c|c|}
\hline & $\underline{\text { ASD - Mean (S.D.) }}$ & $\underline{\text { TD }- \text { Mean (S.D.) }}$ & & \\
\hline & $n=25$ & $n=21$ & test statistic & p-value \\
\hline Age & $34.2(9.6)$ & $32.5(12.4)$ & $t=0.53$ & 0.6 \\
\hline Gender & $23 \mathrm{~J}^{\lambda} ; 2$ ㅇ & 17 ठै; 4 ㅇ & $X_{2}=1.22$ & 0.28 \\
\hline Adaptive Behavior Composite & $77.4(1.8)$ & $100.6(1.9)$ & $t=8.63$ & $<0.0001 * * *$ \\
\hline Communication & $75.8(2.2)$ & $101.4(2.6)$ & $t=7.50$ & $<0.0001 * * *$ \\
\hline Daily Living Skills & $81.0(2.0)$ & $102.8(2.2)$ & $t=7.24$ & $<0.0001 * * *$ \\
\hline Socialization & $79.1(1.8)$ & $101.3(1.5)$ & $t=9.04$ & $<0.0001 * * *$ \\
\hline Motor Skills & $85.7(2.6)$ & $98.5(2.1)$ & $t=3.72$ & $0.0006 * * *$ \\
\hline
\end{tabular}

Table 1: Demographic and adaptive functioning description of the sample. 


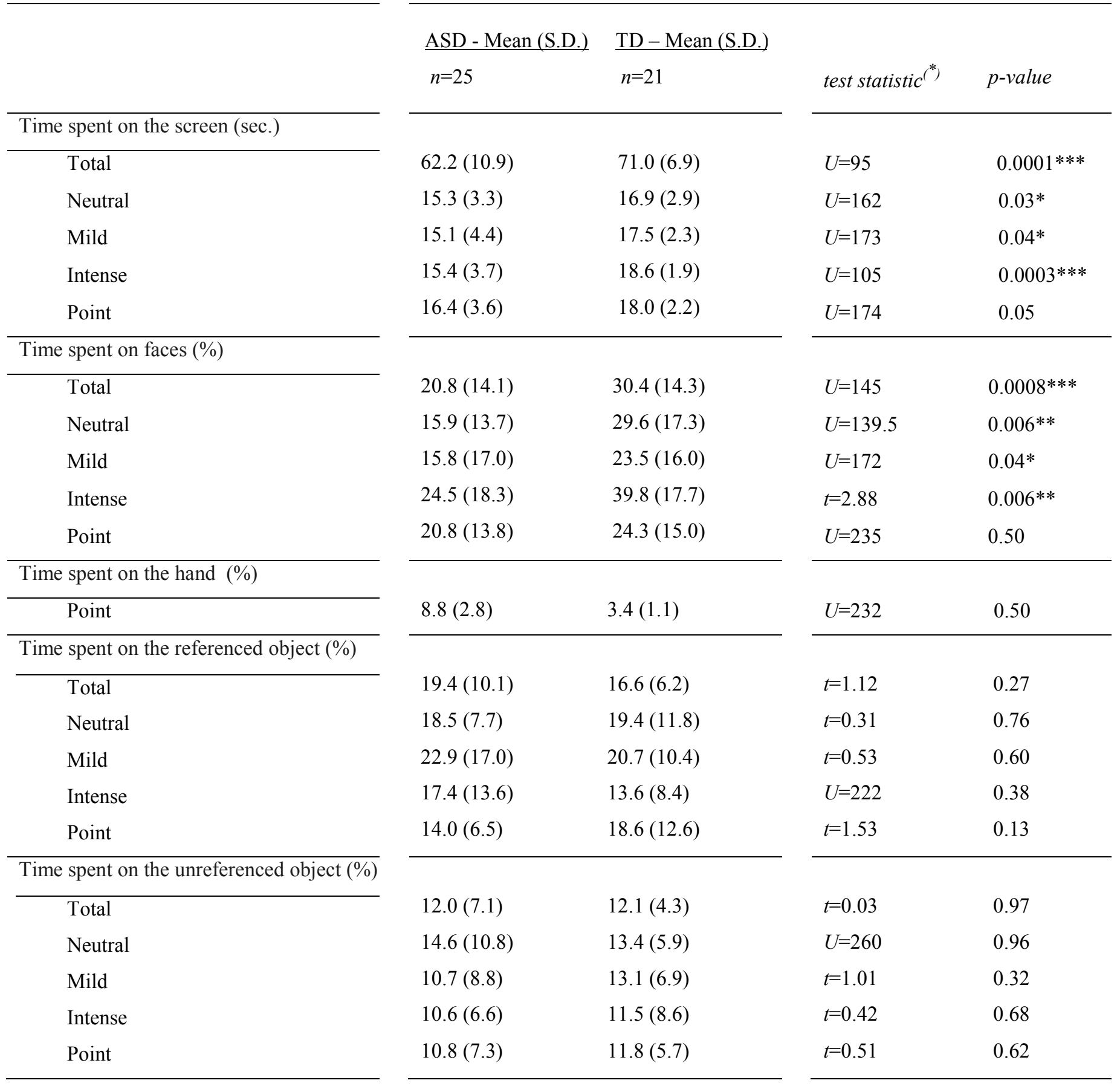

Table 2: Comparison between ASD and TD groups for the dependent variables from the RJA eye-tracking task (time spent on the screen, time spent on faces, time spent on hand, time spent on the referenced object and time spent on the unreferenced object).

${ }^{*}$ ) According to Agostino-Pearson normality test, Student's $t$ test (in case of normal distribution) or Mann-Whitney $U$ test (in case of non-normal distribution) have been applied. 